\title{
Three-Dimensional Field Map of the Fermilab D0 Detector
}

\author{
J. Ostiguy and R. Yamada \\ Fermi National Accelerator Laboratory \\ P.O. Box 500, Batavia, Illinois 60510
}

August 1991

* Presented at the 14th Biennial IEEE Particle Accelerator Conference, San Francisco, CA, May 6-9, 1991. 


\section{Disclaimer}

This report was prepared as an account of work sponsored by an agency of the United States Government. Neither the United States Government nor any agency thereof, nor any of their employees, makes any warranty, express or implied, or assumes any legal liability or responsibility for the accuracy, completeness, or usefullness of any information, apparatus, product, or process disclosed, or represents that its use would not infringe privately owned rights. Reference herein to any specific commercial product, process, or service by trade name, trademark, manufacturer, or otherwise, does not necessarily constitute or imply its endorsement, recommendation, or favoring by the United States Government or any agency thereof. The views and opinions of authors expressed herein do not necessarily state or reflect those of the United States Government or any agency thereof. 


\title{
Three-Dimensional Field Map of the Fermilab D0 Detector
}

\author{
Jean-François OSTIGUY and Ryuji YAMADA \\ Fermilab; P.O. Box 500, Batavia, IL 60510
}

\begin{abstract}
The $\mathrm{D} 0$ detector is a general purpose hadron collider detector presently under construction at Fermilab and scheduled to be put in operation in the fall of 1991 . The D0 muon detection system is composed of three major toroids referred to respectively as the Central Field (CF) toroid and the End Field (EF) toroids. The complete detector weighs in excess of 4000 metric tons and rests on a steel platform. The muon detection system was designed using standard 2D codes and flux maps inside were obtained for each of the toroids taken separately. Various magnetic field measurements were performed; discrepancies with the design calculations have been observed and attributed to three dimensional effects. In this paper, we compare the predictions of the 2D computations to $3 \mathrm{D}$ calculations for a fully assembled detector. We also estimate the electromagnetic forces between the toroids and discuss other 3D effects, in particular, the effect of the supporting platform.
\end{abstract}

\section{Introduction}

The D0 detector (located in the D0 straight section of the Tevatron ring) is a new general purpose hadron collider detector scheduled to be put in operation in the fall of 1991. CDF (Collider Detector at Fermilab), the other major detector (located in the B0 straight section) has been in operation since 1986. Detailed descriptions of the different subsystems which constitute the D0 detector can be found elsewhere [1]. We shall be concerned here with the muon detection system and more specifically with the magnetic aspects of this system.

Muons being the among the most penetrating particles, the muon detector is the outermost of a series of concentric detectors centered on the interaction region. This detector (figure 1) is primarily constituted of iron toroids to deflect the muons and proportional drift chambers to determine their trajectories. There are three principal iron toroids: the central field (CF) toroid, and two end field (EF) toroids. Two smaller toroids, mounted-inside the openings of the EF toroids complete the assembly. These toroids called SAMUS, (for Small Angle MUon Spectrometer) intercept small angle muons.

Three layers (A,B and C) of proportional drift tube chambers are used to gather angular and positional information [2]. Layer A covers the whole inside surfaces of the CF and EF toroids. Layer $B$ is placed just outside the toroids and layer $C$ is separated by $136 \mathrm{~cm}$ from layer $B$. The position and angle of the impinging particle into the toroid iron can be determined with an accuracy of $\pm 0.6 \mathrm{mrad}$ and $\pm 0.1 \mathrm{~mm}$ respectively. For an outgoing particle, the corresponding accuracies are $\pm 0.2 \mathrm{mrad}$ and

\footnotetext{
-Operated by the Universities Research Association, Inc., under contract with the U.S. Department of Energy.
}

$\pm 0.17 \mathrm{~mm}$. The momentum of the muons is determined by deflecting their trajectories in a magnetic field. To this effect, a flux of approximately $1.9 \mathrm{~T}$ circulates in each one of the toroids. The momentum resolution $\delta p / p$ is limited by multiple scattering of the muons in the iron and the measurement error of the muon track. The uncertainty on the transverse momentum due to multiple scattering is independent of the momentum. For the DO CF toroid which is about 1 meter thick, this error is of the order of $10 \%$. The uncertainty due to measurement error of the muon track is typically of the same order and increases linearly with $p$ [2]. A good map of the field in the iron can be obtained from a two-dimensional model. Complications arise when one attempts to use the same 2D model to compute the the fringing flux; in that case, agreement with measurements is not entirely satisfactory. Although the fringing flux has very little effect on the muon trajectories, its presence may have serious consequences. For purely mechanical reasons, the CF coils are not distributed symmetrically between the top and the bottom of the CF toroid. As a result, the fringing field on the beam axis possesses a non-zero transverse component in the orbital plane of the accelerator which may affect the beam trajectory. More importantly, the tunnel harbors, in addition to the (superconducting) Tevatron ring, a second (conventional) ring known as the Main Ring. The Main Ring which is located approximately $207 \mathrm{~cm}$ above the Tevatron in the D0 area, is used to accelerate the protons and anti-protons before they enter the Tevatron; it is also used to accelerate the protons necessary for anti-proton production. Thus, while the protons and anti-protons stored in the Tevatron collide, protons are circulating in the Main Ring. Contrary to the CDF detector where an overpass was built, the Main Ring beam goes right through the D0 detector. It is important to make sure that the transverse magnetic field along the Main Ring beam path will not produce significant perturbations. Aside from the effect it may have on the beam trajectories, the fringing flux may also affect the accuracy of the drift tube chambers located near the toroid surfaces, it may cause the malfunction of certain electronic components, especially transformers. Finally, the fringing flux may affect the performance of the scintillation counters photomultiplier tubes which must be properly shielded.

The D0 detector weighs in excess of 4000 metric tons. It is supported by a steel platform under which a large amount of electronic equipment is located. It is important to understand the fringing fields in this region as well as the effect of the platform on the flux distribution in the toroids. This can only be achieved with a full 3D model. Finally, it is difficult to produce a reliable estimate of the electromagnetic forces acting between the CF and EF toroids without resorting to full 3D analysis. 


\section{D Field Computations}

During the last decade, the advent of relatively inexpensive and powerful computers has rendered practical the development of large scale three dimensional magnetostatics codes. A number of such codes are now commercially available. Most of them, including TOSCA [4], the code we have used to obtain our results, are based on hybrid formulation where the field is separated into solenoidal and irrotational components. The solenoidal component is usually taken to be the field due to the current-carrying conductors and is obtained by straightforward application of Ampere's formula. The irrotational component is represented by a scalar potential which is computed by the finite element method. To avoid numerical instabilities due to cancellation errors, TOSCA can represent the totality of the field by a scalar potential in regions of high permeability.

For a give mesh resolution, three dimensional calculations are significantly costlier than the corresponding two dimensional calculations. Assuming a linear resolution of $N$ nodes, the number of floating point operations required to solve a $3 \mathrm{D}$ problem grows roughly as $C_{1} N^{3}+C_{2} N^{3(1+\alpha)}$ where $\alpha$ is typically 0.3 . The two terms correspond respectively to the number of operations required to calculate the coil field and the number of operations required to solve the finite element equations. When $N$ is reasonably small, the relation between the number of floating point operations and the time required to obtain a solution is linear. As $N$ increases, the storage requirements become larger than the physical memory available and the computer starts moving pages back and forth between physical memory and disk. In practice, by the time the storage requirements have reached about twice the size of the physical memory available, the performance degradation becomes totally unacceptable. All our calculations were performed on a Solbourne Series 5 model 800 workstation with 64 Mbytes of physical memory using TOSCA V6.0. With this setup, the practical limit is about 50,000 nodes (with second order isoparametric elements). For a relatively uniform mesh this corresponds to a linear resolution of roughly $\sqrt[3]{50000} \simeq 37$ nodes. This is barely sufficient. Even when full advantage was taken of the model symmetry, judicious positioning of the nodes was required to maximize accuracy of the results. It is interesting to note that the practical limit on the number of nodes was not determined by storage constraints but rather by the large number of coils (36). A typical run took roughly 30 hours; more than $80 \%$ of the CPU cycles were used to compute the coil fields from Ampere's formula.

\section{Electromagnetic Forces}

The net magnetic force acting on a body can be obtained by integrating the force acting on each differential volume element (in MKS units using the convention $\mathbf{B}=\mu_{0} \mathbf{H}+\mathbf{M}$ )

$$
\mathbf{F}=\int[\mathbf{J} \times \mathbf{B}+(\nabla \times \mathbf{M}) \times \mathbf{B}] d V
$$

In general, the problem of computing the net force acting on a ferromagnetic body is an extremely complex one because the magnetization depends both on the past hiotory and on the state of stress of the body. In practice, one must assume a single valued constitutive relation, independent of the state of stress. It is then possible to express the integrand in equation (1) as the divergence of a tensor (the Maxwell stress tensor) and to transform the resulting expression into a surface integral

$$
\mathbf{F}=\int\left[(\hat{\mathbf{n}} \cdot \mathbf{B}) \mathbf{H}-\frac{1}{2}(\mathbf{B} \cdot \mathbf{H}) \hat{\mathbf{n}}\right] d S
$$

where $\hat{\mathbf{n}}$ is the unit outward normal vector. From a computational standpoint, the principal interest of relation (2) is that it does not involve derivatives of the field. In theory, the integration can be performed on any surface which completely encloses the body, as long as this surface does not enclose any conductors or magnetized matter which are part of another body. The method described above is basically the one used by TOSCA. In the particular case where one is interested in the net force acting on a coil, TOSCA can also perform the integral (1) directly when $\mathbf{M}=0$.

\section{Results}

Due to the symmetry of the detector, it is clear that the magnetic force acting on the EF toroid does not possess any significant $x$ and $y$ components. However, there is a force acting between the CF and EF toroids in the axial direction. To understand the origin of this force, one can reason as follows: each of the 20 coils of the CF toroid has a magnetic image located behind the plane of the EF toroid. Similarly, each of the 8 coils of the EF toroid has an image behind the plane defined by the surface of the CF toroid. Since the image currents are circulating in the same direction as the real currents, the net force is attractive. From equation (2), the $z$-component of the force is

$$
F_{x}=\int\left[\frac{1}{2} H_{z} B_{z}-\left(H_{x} B_{x}+H_{y} B_{y}\right)\right] d S
$$

By integrating over the whole $x-y$ plane at a longitudinal coordinate corresponding to the center of the air gap between CF and EF, we obtain a force of approximately $4.5 \mathrm{kNewtons.} \mathrm{The} \mathrm{force} \mathrm{is} \mathrm{attractive,} \mathrm{as} \mathrm{expected.}$

Figure 2 compares the results of measurements of the horizontal component of the fringing field along a vertical line situated exactly at the center of the CF toroid with the predictions of the 2D model [3] and the full 3D model. The agreement of the 3D calculations with the measurements is obvious. The remaining discrepancies can probably be explained by the presence of three very small air gaps in the CF toroid which have not been included in the $3 \mathrm{D}$ model. To facilitate access to the interior region, the CF toroid is mounted on rollers and separates into two identical parts. Without small gaps in the toroid, it becomes difficult to separate the toroid because of the substantial force associated to the presence of the remanent magnetization.

One of the motivations for performing a full $3 \mathrm{D}$ analysis of the D0 muon system was to study the effect of the presence of the steel base on the flux distribution in the CF toroid. More specifically we were concerned with the flux circulating between the lower portion of the CF toroid and the steel base. A large amount of flux leaking into the base can lead to a non-uniform flux distribution in the CF 
toroid. As a first approximation, the base has been modeled as a solid piece of iron. In reality, the base is hollow and is made of iron plates approximately $6 \mathrm{~cm}$ thick. Figure 3 compares the flux density in the center of the bottom portion of the CF toroid in the presence and in the absence of the supporting structure. As expected, the presence of the base causes a $2 \%$ decrease of the flux in CF, which is probably tolerable. Note that the field at both extremities of CF is weaker than in the center. The effect is barely noticeable in the absence of the base and becomes a lot more pronounced in its presence.

\section{Conclusion}

Considering the limitations on the spatial resolution of the mesh, the field map produced by a 3D finite element code appears to be accurate enough to be useful. The maps which we have been generated will be used to fine-tune the D0 detector. We also plan to use $3 \mathrm{D}$ analysis to examine a certain number of problems posed by a proposed upgrade of the detector. The upgrade would consist mainly in adding a solenoid inside CF to produce an axial field in the interaction region.

\begin{tabular}{|l|r|r|r|}
\hline Dimensions & CF Toroid & EF toroids & SAMUS \\
\hline \hline Longitudinal $(\mathrm{cm})$ & 760 & 150 & 150 \\
\hline Horizontal $(\mathrm{cm})$ & 860 & 860 & 170 \\
\hline Vertical (cm) & 850 & 850 & 170 \\
\hline Weight (mton) & 2000 & 800 & 32 \\
\hline Number of colls & 20 & 8 & 4 \\
\hline turns/coil & 10 & 8 & 24 \\
\hline Current $(A)$ & 2500 & 2500 & 417 \\
\hline
\end{tabular}

\section{References}

[1] "The D0 Experiment at the Fermilab Anti-protonProton Collider" Design Report, Fermilab (1984)

[2] K. Kleinknecht, "Detector for Particle Radiation", Cambridge University Press 1986

[2] C. Brown et al., "DO Muon System with Proportional Drift Tube Chambers", Nucl. Instr. and Meth. A279 331-338 (1989)

[3] R. Yamada, "The Magnetic Design and Field Measurement of Fermilab Collider Detectors: CDF and D0",Proceedings of the 11 th International Conference on Magnet Technology, Tsukuba, Japan (1989).

[4] J. Simkin and C. W. Trowbridge, "Three-dimensional Nonlinear Electromagnetic Field Computations, using Scalar Potentials", IEE Proc.,127,6, 368-374 (1980).

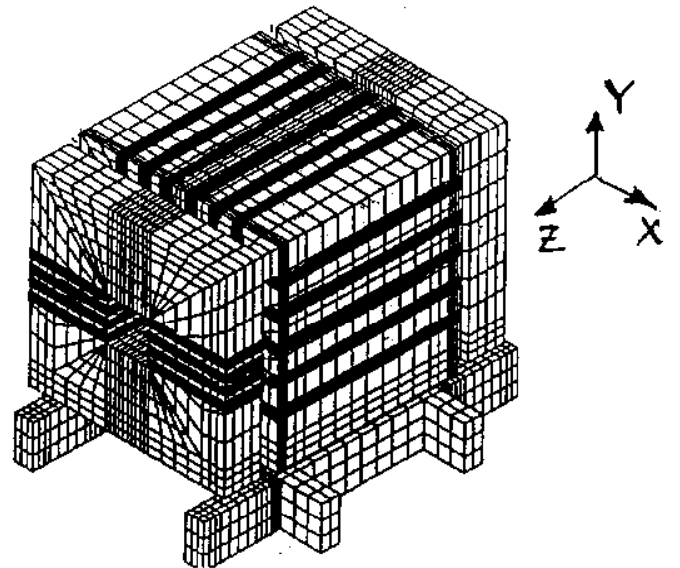

Figure 1: A perspective view of the D0 toroids. The SAMUS toroids have not been included in the model and are therefore not illustrated.

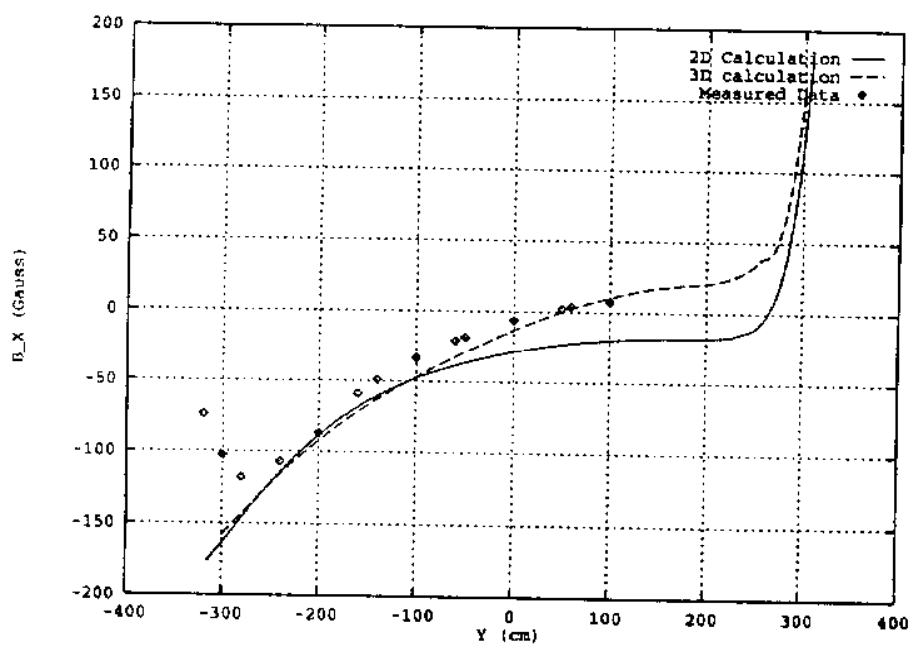

Figure 2: Horizontal component of the fringing field inside the CF toroid along the vertical axis.

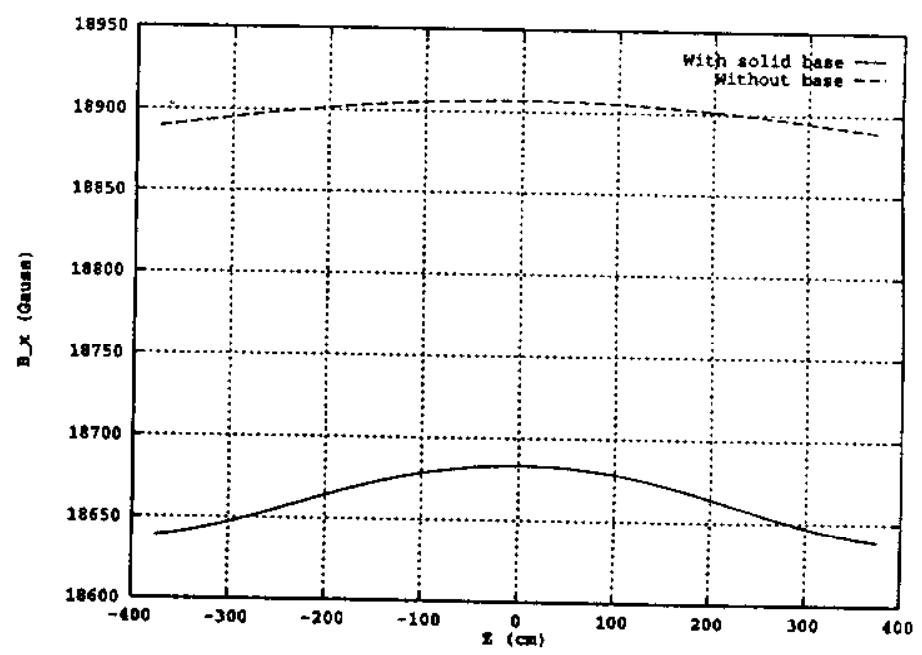

Figure 3: Magnitude of the flux in the base of the CF toroid. 